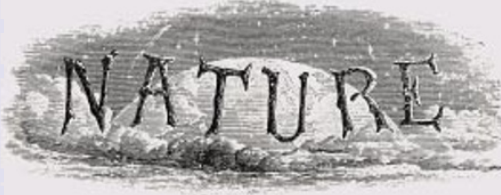

100 YEARS AGO

The Daily Chronicle recalls that a London paper of the first week of $\mathbf{1 8 0 0}$ alluded to the then recent hot disputes in France and England respecting the beginning of the nineteenth century. According to the paragraph, the famous Joseph Jérôme Lefrançais de Lalande, who then occupied the Chair of Astronomy in the University of Paris, had taken an active part in the controversy, and he had pronounced in favour of January 1,1801 . His decision had been generally accepted as correct on both sides of the Channel. The newspaper referred to remarks: "The same ridiculous question was agitated in 1700." So does history repeat itself.

From Nature 4 January 1900.

\section{YEARS AGO}

For some ten years, at the Zi Ka Wei Observatory, in Shanghai, China, we have been using, with exceptional success, a new technique in our weather forecasting. This method is based on an as yet unexplained correlation between the usual ionosphere echoes $\left(E, F\right.$ and $\left.F_{2}\right)$ and the future movement or behaviour of the three main air masses, which make the weather all over the world: polar, maritime and tropical, or equatorial, as some people call it. Our results have already been published in the Bulletin of the Meteorological Society of America, in 1946, and in a paper sent to the last Pacific Science Congress held in New Zealand last February. We found, in 1939, in Zi Ka Wei that by pulsing on the mean critical frequency for the $E$-layer at our station (6 Mc.), any day of the year, after sunrise and before sunset, we could forecast what type of air mass would make the weather over an area of $\mathbf{4 0 0} \mathbf{~ k m}$. around Shanghai. For example, we found that when we had an E-echo, the maritime (Pacific Ocean) air mass would either stay over us, if it was already there, or it would come over us, if at the time of pulsing another type of air mass was covering our regions. When we obtained an $F$-echo, the polar (Siberian) air mass would make the weather, while an $F_{2}$-echo meant the arrival of the tropical air, or its staying if already there. Knowing by this technique the type of air mass due to make the weather, we could forecast its future characteristics, dry or damp, overcast or clear, windy or calm, good or bad visibility, high or low temperatures, etc.

From Nature 7 January 1950. of which provides a measurement of the turbulence in a different direction. The computer-intensive task of converting these linear projections into the original threedimensional profile is known as atmospheric tomography.

Ragazzoni et al. ${ }^{1}$ have shown that the tomographic method works. They collected optical-distortion measurements from four stars in the constellation Aquila, and used least-squares techniques to predict atmospheric distortions for the central star as a linear combination of the other three measurements. The mean-square error in this prediction is 17 times smaller than the initial distortion, and three times smaller than the error obtained when the leastsquares method is replaced by an arithmetic average. Two deformable mirrors could use these predictions to correct for distortions in real time for the entire constellation. The inferior results obtained by averaging are comparable to what could be obtained with a single mirror and guide star.

Studies to assess the feasibility of MCAO for existing telescopes are already underway. For an 8-metre telescope, a system with three mirrors and five laser guide stars would mean a ten-fold increase in the area of the compensated field-of-view compared with conventional adaptive optics, and would dramatically improve the uniformity of correction within this area. The individual mirrors and optical-distortion sensors required to set up such a system are already available. Existing lasers are not yet up to the mark, but are being developed in the right direction. Calculations in real time would need to increase up by a factor of 15 to make atmospheric tomography possible, but processing on this scale has been demonstrated by other adaptive optics programs. The use of MCAO for solar astronomy is also under investigation. In this case, guide stars are not needed, because optical distortions can be determined from images of the granulation in the solar photosphere ${ }^{8}$.

In the longer term, MCAO may be an important technology for the much larger 30 - to 100 -metre ground-based telescopes currently under discussion ${ }^{9}$. How the number of guide stars and deformable mirrors will scale with telescope size is not well understood, but it may be that the maximum angular separation allowed between guide stars for effective tomography is proportional to telescope diameter. If so, the relatively low angular density of bright natural stars would suffice for atmospheric tomography with 100-metre telescopes, eliminating the need for lasers. Of course, the technical advances needed to develop deformable mirrors and optical-distortion sensors for telescopes of this size are considerable. Getting to grips with the possibilities and requirements of such systems will be an important task for the adaptive optics community in the years ahead.

Brent Ellerbroek and François Rigaut are at the Gemini Observatory, 670 North A'ohoku Place, Hilo, Hawaii 96720, USA.

e-mail:bellerbroek@gemini.edu

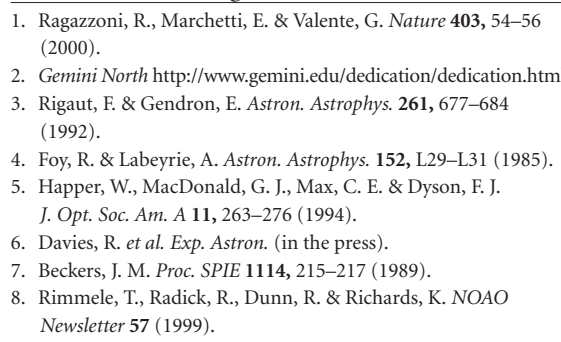

9. Gilmozzi, R. et al. Proc. SPIE 3352, 778-791 (1998).

\title{
Living on the edge
}

\section{Thomas Brooks}

$\mathrm{n}$ an undisturbed ecosystem, populations at the edges of species' ranges are less dense and more variable in space and time than at their centres. So they are more prone to extinction ${ }^{1}$. But surprisingly, accumulating evidence suggests that edges are where the last remaining populations of many species survive $^{2,3}$. On page 84 of this issue, Channell and Lomolino ${ }^{4}$ extend their previous work on this apparent paradox. For 245 species across a wide variety of taxonomic groups and geographical regions, they show that the collapse of ranges towards the periphery is the rule, not the exception. Their explanation is that human impacts, such as deforestation, moving in a front across the landscape, simply overwhelm the underlying geographical ecology. Many species have life on the edge thrust upon them.

Channell and Lomolino chose 245 species for which maps of current and historical ranges are available. For each they located the centre point in a species' historical range as that most distant from any range edge. They defined each species' range periphery as everywhere more than half this distance away from its centre. They then calculated a species' 'index of centrality' as the proportion of its recent range that falls within the central portion of its historical range. Two-thirds of their species survived preferentially in their historical peripheries, and one-third only in these peripheries. The pattern of survival on the edge is a general one. 
An example is the black-footed ferret (Mustela nigripes, see Fig. 1 on page 84), which now survives only in the northwest corner of its historical range.

How much trust can we put in the estimates of historical ranges? Mapping the contemporary ranges of species is hard ${ }^{5}$. So too is documenting past extinctions, as the work of the Committee on Recently Extinct Organisms $^{6}$ exemplifies. Assessing historical distributions combines these two difficulties. Historical ranges will tend to be less finely resolved, and will approximate the extent of a species' occurrence rather than its area of occupancy ${ }^{5}$. They will therefore appear less fragmented than current ranges. (This problem is scale dependent, because it is easier to map the ecologically marginal areas of a species' range at fine scales.) This should not alter Channell and Lomolino's indices of centrality, however, because the effect of increasing the proportion of historical periphery should be balanced by the effect of the increasing proportion of current range within this periphery.

There are two places that show no tendency for peripheral populations to survive. The first, Hawaii, illustrates a case of range implosion, where threats simultaneously affected all of the coastal edges of species' ranges, pushing their last populations into the central uplands. In fact, Hawaii may not even be an exception, if considered from the perspective of ecological occupancy as well as geographical extent. Olson and James ${ }^{7}$ have suggested that before humans arrived many Hawaiian species were actually confined to the lowlands, and so had doughnut-shaped ranges. To the last remnants of Hawaii's animals and plants, the uplands may be the ecological periphery of their ranges, even if they are the geographical centre.

Africa is the other exception where species show no significant tendency to survive at the edges of their ranges. Channell and Lomolino's explanation is that people have a longer history in Africa than anywhere else ${ }^{8}$. Thus, human activity is already distributed throughout the landscape, instead of moving across it by contagion. This cannot be the whole explanation, however, because the declines of all of Channell and Lomolino's continental species are recent, rather than stemming from first human contact. (By contrast, the Pacific islands in their study were reached by people only in the last two millennia ${ }^{7}$.)

An alternative possibility is suggested by the taxonomic composition of the data. Nearly all (20 out of 24) of the African species are large mammals, compared with less than half in other regions. Human hunters prefer large species ${ }^{8}$, and so have caused these range collapses in Africa. Elsewhere, the examples of extinction documented by Channell and Lomolino are usually the result of habitat destruction and
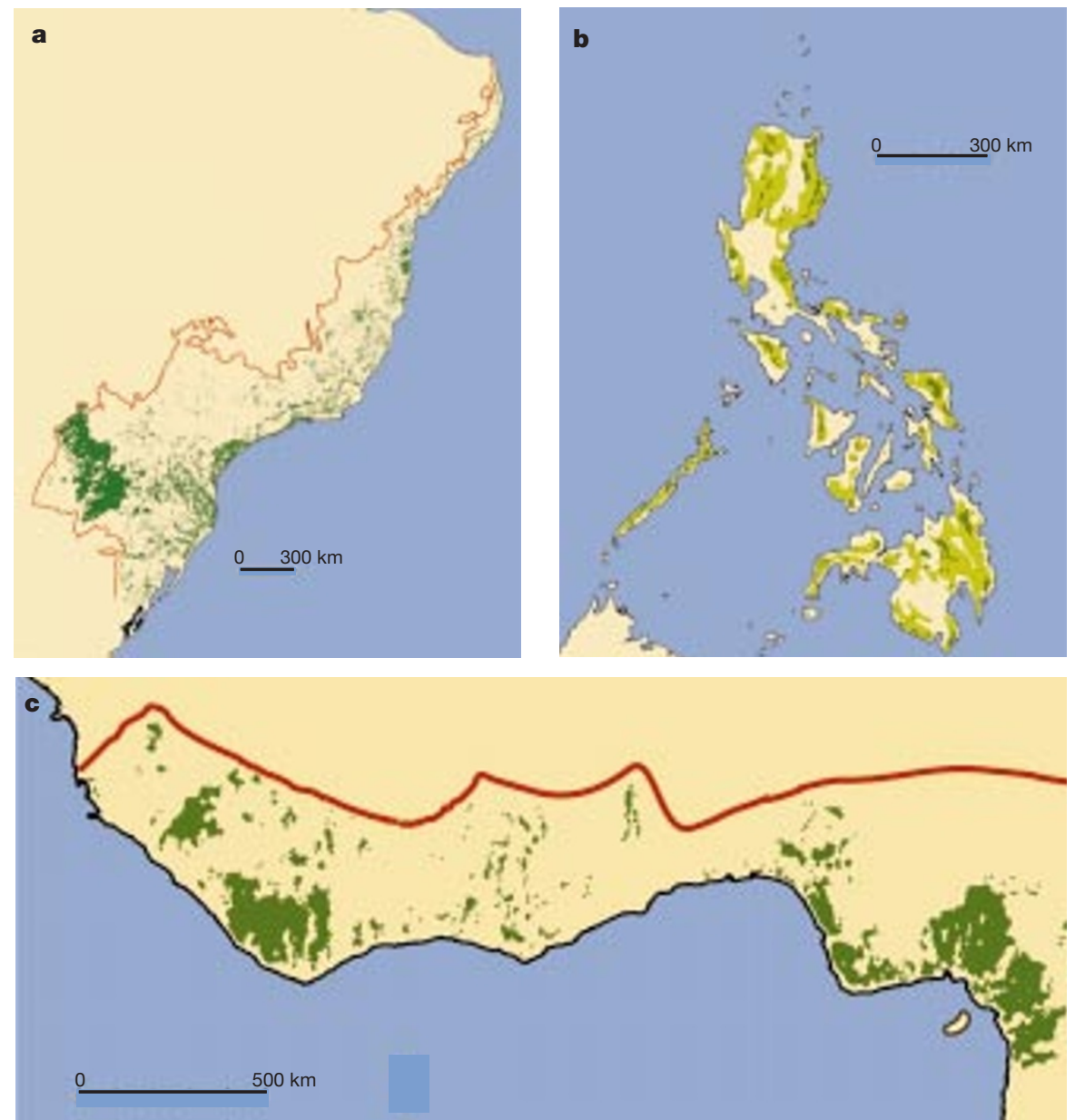

Figure 1 The extent of current and historical habitat in three 'hotspots' ${ }^{10}$. Surviving habitat tends to lie in the historical periphery. a, The Serra do Mar and the Paraná basin of the South American Atlantic forests (red line, historical range; green, surviving habitat). b, The northern Luzon and southern Mindanao in the Philippines (light green, historical range; dark green, surviving habitat). c, The extreme west and east of the Guinea region of West Africa (red line, historical range; green, surviving habitat).

invasive species. Could overhunting drive populations inwards, whereas other threats move laterally across the landscape? This suggestion is eminently testable by comparing the pattern of range collapse with body size of the species concerned or (more directly) with the human threat at work in the landscape.

Channell and Lomolino's work ${ }^{4}$ is obviously relevant to individual species. But how might their conclusions be important for broader planning, given that we have neither the resources nor the time to conserve species one-by-one ${ }^{9}$ ? Numerous species have overlapping distributions, such as the many taxa - maybe half of all the world's species - that co-occur in 25 'hotspots' that together make up only $1.4 \%$ of land area ${ }^{10}$. Data for these species are scarce, but patterns of habitat loss should match the geographies of their collapsing ranges ${ }^{11}$. Based on Channell and Lomolino's results, we would expect disproportionate amounts of habitat to survive on the peripheries of the hotspots. This appears to be the case (Fig. 1$)^{10}$. Should attempts to conserve these areas be concen- trated on their edges? On average, the answer is 'no's, because these geographically marginal areas will also tend to be ecologically marginal. Within the entire periphery of any one hotspot, though, there are likely to be areas that are particularly worth conserving, thanks to their isolation from the habitat loss driving extinction across the region.

Channell and Lomolino conclude that "the geography of recent extinctions" - by which they mean local extinctions - "is largely a geography of humanity". The geography of global extinctions, however, is also that of the hotspots ${ }^{12}$. Within hotspots we cannot afford to ignore the lesson that peripheral sites, where biological diversity has been forced to live on the edge, may now hold some of the last opportunities for conservation.

Thomas Brooks is at the Zoological Museum, University of Copenhagen, the Department of Zoology, University of Cambridge, and the Center for Applied Biodiversity Science, Conservation International, 2501 M Street NW, Suite 200,

Washington DC, 20037, USA.

e-mail:t.brooks@conservation.org 
1. Curnutt, J. L., Pimm, S. L. \& Maurer, B. A. Oikos 76, 131-144 (1996).

2. Lomolino, M. V. \& Channell, R. J. Mamm. 76, 335-347 (1995).

3. Lomolino, M. V. \& Channell, R. Conserv. Biol. 12, 481-484 (1998).

4. Channell, R. \& Lomolino, M. V. Nature 403, 84-86

(2000).

5. Gaston, K. J. Ecography 17, 198-205 (1994).

6. http://www.creo.org

7. Olson, S. L. \& James, H. F. in Quaternary Extinctions: A Prehistoric Revolution (eds Martin, P. S. \& Klein, R. G.) 768-780 (Univ. Arizona Press, Tucson, 1984).
8. Alroy, J. in Extinctions in Near Time: Causes, Contexts, and Consequences (ed. MacPhee, R. D. E.) 105-143 (Kluwer/Plenum, New York, 1999).

9. Williams, P. H. in Conservation in a Changing World (eds Mace, G. M., Balmford, A. \& Ginsberg, J. R.) 211-249 (Cambridge Univ. Press, 1998).

10. Mittermeier, R. A., Myers, N., Robles Gil, P. \& Goettsch Mittermeier, C. Hotspots (CEMEX, Mexico City, 1999).

11. Brooks, T. M., Pimm, S. L. \& Collar, N. J. Conserv. Biol. 11, 382-394 (1997).

12. Pimm, S. L. \& Brooks, T. M. in Nature and Human Society: The Quest for a Sustainable World (ed. Raven, P.) 38-54 (Natl Acad. Press, Washington DC, 1999).

\section{Apoptosis}

\section{Caspases find a new place to hide}

Huseyin Mehmet

$\mathrm{T}$ he philosophical muse proclaiming that "death is the essential condition of life" has a firm footing in biology, for programmed cell death (apoptosis) is obligatory for normal development of multicellular organisms ${ }^{2}$. But apoptosis is a double-edged sword, and deregulated cell death is implicated in a growing number of clinical disorders $^{3}$. So, the apoptotic process needs to be tightly regulated. One way in which this is done is by physically segregating the different components of the apoptotic machinery - only when the death switch is tripped are the tools of execution brought together in the cytosol and the suicide programme activated.

The two main compartments known to be involved in such segregation are the plasma membrane, where both death and survival receptors reside, and the mitochondrion, which is home to several proteins that regulate apoptosis. Now, reporting on page 98 of this issue, Yuan and colleagues ${ }^{4}$ point to the endoplasmic reticulum as a third subcellular compartment implicated in apoptotic execution. Furthermore, they provide evidence linking activation of a hitherto obscure apoptotic enzyme, caspase-12, to Alzheimer's disease.

Famous mainly for the synthesis and processing of secreted proteins and the storage of intracellular calcium, the endoplasmic reticulum has already provided us with clues as to its alter ego. First, both pro- and antiapoptotic members of the Bcl-2 family are located in intracellular membranes including the nuclear envelope, the outer mitochondrial membrane and the endoplasmic reticulum ${ }^{5}$. And second, cytosolic $\mathrm{Ca}^{2+}$ has been implicated as a pro-apoptotic second messenger ${ }^{6}$ involved in both triggering apoptosis and in regulating death-specific enzymes.

Among the most prominent of these death-specific enzymes is a family of cysteine-dependent aspartate-specific proteases known as the caspases ${ }^{7}$. These enzymes can be broadly divided into two groups: initiator caspases (such as caspase- 8 and caspase-9) whose main function is to activate downstream caspases, and executor caspases (such as caspases-3, -6 and -7), which are responsible for dismantling cellular proteins. The two main apoptotic pathways the death receptor and mitochondrial pathways - are activated by caspase- 8 and caspase-9, respectively, both of which are found in the cytoplasm (Fig. 1). Caspase- 8 is recruited to a death-inducing signalling complex only when death receptors such as
Fas or the tumour-necrosis factor receptor are oligomerized after binding of specific ligands. In contrast, caspase- 9 is activated when cytochrome $c$ is released into the cytoplasm from the space between the inner and outer mitochondrial membranes.

Yuan and co-workers ${ }^{4}$ now show that another caspase, caspase-12, localizes not to the cytosol but to the endoplasmic reticulum. Caspase-12 is specifically involved in the apoptosis that results from stress in the endoplasmic reticulum. Treatment with compounds such as brefeldin A (which inhibits transport from the endoplasmic reticulum to the Golgi body) or tunicamycin (which inhibits $N$-glycosylation in the endoplasmic reticulum) triggers activation of caspase-12. But the strongest activation is seen in response to thapsigargin, which disrupts intracellular $\mathrm{Ca}^{2+}$ homeostasis, or to the $\mathrm{Ca}^{2+}$ ionophore A23187. Apoptosis triggered through pathways that do not involve the endoplasmic reticulum, such as serum deprivation or Fas activation, do not result in activation of caspase- 12 .

To study the localization of caspase-12, Yuan and colleagues used a specific caspase12 antibody in immunoblotting experiments and detected bands in a fraction of brain extract that also contained an endoplasmic-reticulum-specific protein called TRAP $\alpha$. Immunocytochemistry then revealed the perinuclear distribution of cas-

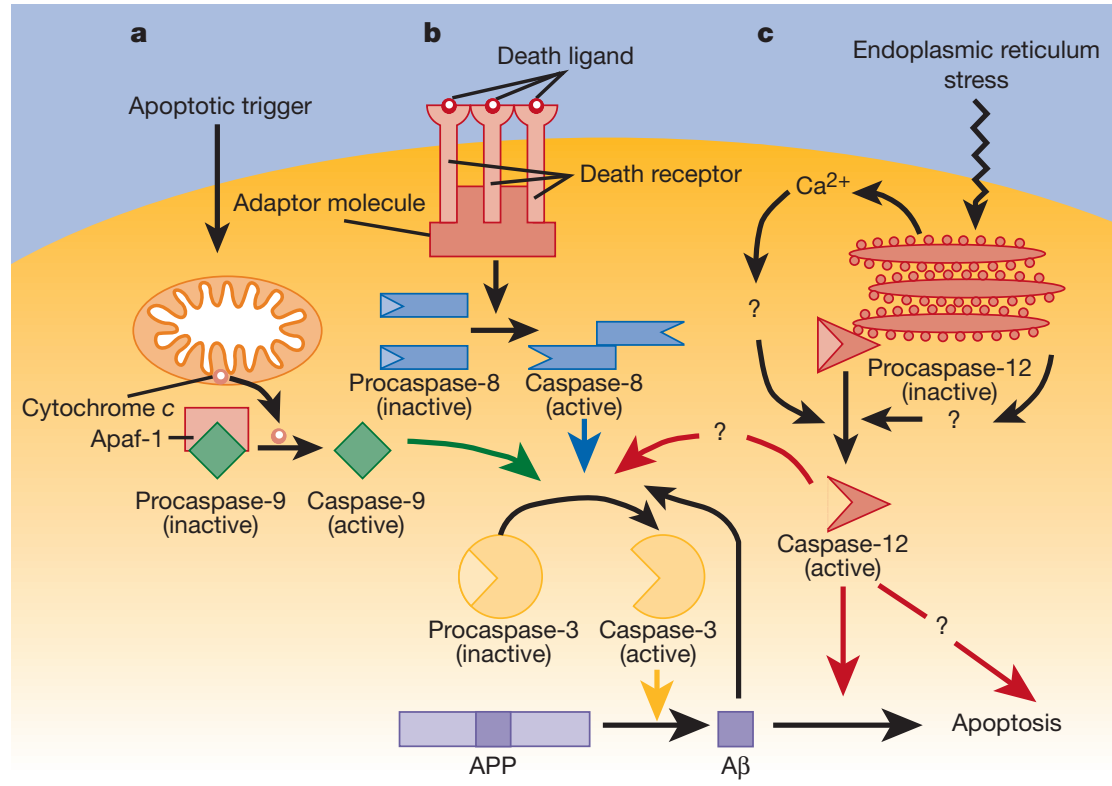

Figure 1 Three distinct apoptotic signalling pathways. a, When the mitochondrion receives appropriate apoptotic cues, or is irreversibly damaged, pro-apoptotic molecules such as cytochrome $c$ are released into the cytosol. Together with ATP, cytochrome $c$ forms a complex with Apaf-1 and procaspase-9, which is released in an active form. b, Oligomerization of death receptors (by specific death ligands) recruits adaptor molecules involved in activation of caspase- 8 . The active caspase is formed from two procaspase- 8 molecules. $c$, After stress to the endoplasmic reticulum, including the release of $\mathrm{Ca}^{2+}$ from intracellular stores, caspase-12 is activated. Activated initiator caspases, such as caspase- 8 and caspase- 9 , activate executioner caspases, including caspase-3. Active caspase- 3 cleaves the $\beta$-amyloid precursor protein (APP), resulting in increased production of amyloid $\beta$-peptide (A $\beta$ ) which can feed back into caspase- 3 activation and execute apoptosis in a caspase-12-dependent manner. The question marks denote possible, but unconfirmed, pathways. 